\title{
Soluble Polysulphide Sorption using Carbon Nanotube Forest for Enhancing Cycle Performance in a Lithium Sulphur Battery
}

\author{
Kai Xi, ${ }^{a, \neq}$ Bingan Chen, ${ }^{b, c, \neq}$ Huanglong Li, ${ }^{b, d}$ Rongsi Xie, ${ }^{b}$ Chenlong Gao, ${ }^{a}$ Can Zhang, ${ }^{b, *}$ \\ R. Vasant Kumar, ${ }^{a, *}$ and John Robertson ${ }^{b, *}$ \\ ${ }^{a}$ Department of Materials Science and Metallurgy, University of Cambridge, Cambridge, CB2 3QZ, UK. \\ ${ }^{b}$ Department of Engineering, University of Cambridge, Cambridge, CB3 OFA, UK. \\ Present address: \\ ${ }^{c}$ AIXTRON Ltd., Anderson Road, Buckingway Business Park, Swavesey, Cambridge, CB24 4FQ, UK \\ $10{ }^{d}$ Optical Memory National Engineering Research Center, Department of Precision Instrument, Tsinghua University, Beijing 100084, China
}

$\ddagger$ both authors contributed equally to this work

\begin{abstract}
15 The rapid capacity decay of lithium-sulphur batteries has been a significant obstacle for practical application, which is generally considered to arise from dissolution of lithium polysulphide in the electrolyte and diffusion away from the cathode. As the lithium content in the polysuphide inceases with further discharge, capacity decay occurs also from the passivating effects by the formation of insoluble sulphides, further amplified by volume increase. More recently, weakening of sulphur adhesion to carbon

20 with progress in discharge is also an important factor in the sulphur cathode degradation. In order to overcome capacity decay caused by all the above mechanisms, we have prepared a composite cathode made of sulphur and high density carbon nanotube (HD-CNT) forest scaffold that is able to interfacially adsorb and volumetrically confine the polysulphide species and accommodate the expansion of sulphur discharge products effectively. This cathode demonstrates very high electrochemical stability and high

${ }_{25}$ discharge capacity up to 200 full discharge/charge cycles even with the use of the basic organic ether electrolyte where polysulphide shows high solubility, thus providing evidence for confinement and interfacial contact. Retention and surface adsorption favoured by minimising the wall-to-wall distance between the aligned CNTs arising from a decrease in the reaction energy of the adsorption. Computational simulation of the interface between polysulphide species and carbon nanotube surface 30 provides first-principles confirmation of improved binding between $\mathrm{C}$ and $\mathrm{S}$ in the polysulphides as wallto-wall distance is decreased. The HD-CNT scaffold is self-binding and highly-conducting thus the conventional additives of binder and carbon black are also fully eliminated. A high discharge capacity of $812 \mathrm{mAh} \mathrm{g}^{-1}$ of sulphur (corresponding to $503 \mathrm{mAh} \mathrm{g}^{-1}$ of the whole cathode material mass) is stably retained after 200 cycles at $400 \mathrm{~mA} \mathrm{~g}^{-1}$ with a small average capacity decay of only $0.054 \%$ per cycle on

35 average These encouraging results provide novel approaches to designing and fabricating long cycle life cathode in a lithium sulphur battery.
\end{abstract}

Kewords: lithium-sulphur battery, cathode, carbon nanotube, polysulphide sorption, first-principles calculation, cycle performance

40

*Corresponding author. Fax: ; Tel: +44 (0)1223 334327; +44 (0)1223 334567.

Email address: can.zhang@ cantab.net (C. Zhang),

rvk10@cam.ac.uk (R.V. Kumar), jr214@cam.ac.uk (J. Robertson)

45

\section{Introduction}

Lithium-sulphur batteries, which use sulphur (S) as cathode and lithium as anode, have attracted considerable attention because of their high theoretical capacity of $1675 \mathrm{mAh} \mathrm{g}^{-1}$ of 50 elemental sulphur and high nominal theoretical energy density of $2500 \mathrm{Wh} \mathrm{kg}^{-1}$.[1-5] Sulphur is readily available particularly as a by-product of oil and natural gas desulfurization and poses less environmental risk than heavy metal based $\mathrm{Co}, \mathrm{Mn}$ compounds or the phosphates that are presently used in Li-ion batteries. ${ }_{55}$ Therefore, sulphur is considered to be a very promising future

cathode material for high energy-density rechargeable batteries. Despite its promising attributes, the Li-S battery is still plagued by various problems, and a rapid capacity fading with cycling is the key issue hindering its widespread practical utilization. ${ }_{60}$ Capacity degradation is ascribed to a combination of factors. The lithium polysulphides as they form during discharge can readily dissolve and diffuse in the electrolyte away from the cathode redox sites. [6] The lithium polysulphides are intermediate products of the electrochemical reduction of sulphur in the 65 organic electrolyte. They can dissolve and diffuse from the cathode to the metallic lithium anode (and also in the reverse 
direction) causing parasitic reactions, which result in lithium anode corrosion, loss of active sulphur and low columbic efficiency. As reduction progresses, the lithium polysulphides will finally result in the precipitation of insulating $\mathrm{Li}_{2} \mathrm{~S}$ and other 5 insoluble compounds on the cathode surface, which inhibit transport of the lithium ions and reduce the battery capacity especially with cycling. The solid sulphides occupy $80 \%$ more volume than sulphur and thus enhance the passivating effect. ${ }^{7}$ Any unutilized sulphur can also have passivating effect due to its 10 insulating property.

To address these issues, some forms of carbon are usually added to the cathode due to their high conductivity, low weight and ready availability in porous structure.[7-12] But the capacity fading is still observed in the basic sulphur/carbon composite 15 systems. Poor interfacial contact degradation due to the $\mathrm{Li}_{x} \mathrm{~S}$ detachment from the carbon surface during the discharge process has been shown recently to be an important issue.[13] Zheng et al [13] recently proved that despite tendency towards volume expansion, weakening of $\mathrm{C}-\mathrm{S}$ bonding with increasing lithium in 20 the polysuphides can result in shrinking away of $\mathrm{S}$ from the $\mathrm{C}$ surface. Thus the interfacial contact between the lithium polysulphides and carbon, crucial for sustaining the redox reaction is degraded, inevitably leading to the formation of aggregated insoluble sulphides. Zheng et al [13] added PVP to 25 electrolyte and used ex-situ TEM to show evidence for improving the C-S bonding.

Most approaches reported in the literature have tended to consider additives that can further limit the solubility and diffusivity of polysulphides in the electrolyte. Just as the above30 mentioned work by Zheng et al [13], who have aimed to avoid loss of binding between $\mathrm{C}$ and the $\mathrm{S}$ products in the electrolyte. Oxides sulphide captures, such as mesoporous silica[14], metal-organic framework (MOF)[10, 15] and titania[16], have been introduced to improve the performance of the sulphur 35 cathode. Several groups also demonstrate that the modification of sulphur electrode by polar polymer additives is consistently shown to improve the cycling performance.[7, 13, 17] In further improve the cycle stability, several precise structural innovations have been proposed, including the use of core-shell morphology, $40[18,19]$ the graphite/lithium metal as a hybrid anode[20] and the introduction of an ion selective membrane[21]. Furthermore, the new electrolyte system based on an organic ether, such as dioxolane (DOL) and 1,2-dimethoxyethane (DME), as a solvent is developed because the carbonate solvents typically used in a 45 lithium-ion battery are usually not suitable for Li-S batteries.[22] The rapid capacity fading arises from the dissolution of lithium polysulphides $\left(\mathrm{Li}_{2} \mathrm{~S}_{\mathrm{n}}, \mathrm{n}=3-8\right)$ into the organic ether electrolyte during the discharge-charge process. Finally, the large volume expansion due to the difference in density between sulphur and 50 the solid lithium sulphide products coupled with the polysulphide dissolution will further accelerate the capacity fading during cycling. Therefore, $\mathrm{LiNO}_{3}$ additive has been introduced to stabilize the Li-metal surface. Meanwhile, the eletrolyte with high $\mathrm{Li}$ salt concentrations has been used to reduce the dissolution of 55 polysulphide by the common ion effect.[23-25] However, the additives will decrease the overall capacity of the battery and introduce additional costs. In this paper, we have focused on a solution that can improve the interfacial binding between $\mathrm{C}$ and the the Li polysufides through morphological control alone 60 without any additives. In order to prove the concept we have intentionally used an electrolyte in which the polysuphides are very soluble.

Recently, carbon nanotube (CNT) has been combined with sulphur to form a cathode for lithium-sulphur battery, thus 65 utilizing CNT's excellent electrical, thermal, and mechanical properties.[26-32] To further constrain the dissolution and the diffusion of polysulphides during the redox reaction, threedimensional (3-D) CNT networks were trialled, based upon vertical aligned carbon nanotube/sulphur composites[29, 30] and 70 hierarchical architecture S/MWCNTs nano/micro-spheres[28]. These 3-D CNT networks provide a new cathode architecture design in the development of lithium-sulphur batteries.

In this work, we find that good cycling stability can be achieved in organic ether electrolyte by effective material 75 structure design rather than incorporating further additives in the carbon/sulphur cathode or the electrolyte. Herein, we show how to make and control a sulphur/CNT array (S/CNT array) composite cathode within a stable and resilient CNT network formation, so that the resulting $\mathrm{Li}-\mathrm{S}$ battery can achieve an 80 electrochemical stability of up to 200 cycles with high discharge capacity retention. Good electrochemical performance could be attributed to the presence of an array of high-density CNT scaffolds with orderly structure that can adsorb the polysulphides and accommodate the volume expansion of sulphur within the

85 basic organic ether eletrolyte. To further understand how the battery performance is influenced by the carbon structure, we carried out first-principles calculations, which shows that a small wall-to-wall distance is favorable for achieving large negative reaction energy of the adsorption. Both the experimental results 90 and the computer simulation provide cross-confirmation for the hypothesis that a well-designed high density nanotube forest is favorable for achieving stronger polysulphide adsorption and ultimately the cycle stability is improved. While successfully addressing the capacity decay problem, additional capacity is 95 available from this novel cathode structure, from the ability to eliminate additional components such as conducting additives (e.g. carbon black) and binders (e.g. polytetrafluoroethene: PTFE) from the cathode system.

\section{${ }_{100} 2$ Material and methods}

\subsection{Cathode Preparation}

A copper foil (50 $\mu \mathrm{m}$ thick, Advent Research Materials Ltd) was used as the substrate for CNT growth. It was cleaned by washing with acetone, IPA and de-ionized water. This was followed by 105 coating the $\mathrm{Cu}$ surface with a $10 \mathrm{~nm} \mathrm{Al}$ layer and a $1.3 \mathrm{~nm} \mathrm{Fe}$ layer by sputtering. The coated foil was then loaded into a chemical vapor deposition system as a substrate for CNT growth [33-38]. The sample was heated up to the CVD temperature in 3 minutes under $500 \mathrm{sccm} \mathrm{H}_{2}$ flow at a pressure of 15 mbar. CNTs 110 were grown at $700{ }^{\circ} \mathrm{C}$ with a $\mathrm{H}_{2} / \mathrm{C}_{2} \mathrm{H}_{2}$ gas mixture by adding acetylene to the flowing hydrogen gas $(500 \mathrm{sccm}$ total gas flow, $2-10 \% \mathrm{C}_{2} \mathrm{H}_{2}$ ). The pressure during the CVD was varied between 1-15 mbar.

Elemental sulphur powder and as-prepared CNT forests were 
dried at $80{ }^{\circ} \mathrm{C}$ under vacuum before use. Then, $0.1 \mathrm{~g}$ sulphur was dissolved completely in $30 \mathrm{~mL}$ toluene (Sigma-Aldrich, $99.8 \%$, anhydrous) solution. The sulphur/CNT cathode was prepared by infiltration of sulphur from the solution into the CNT arrays. 5 More specifically, a sulphur solution was dropped onto the CNT arrays and then dried after 5 minutes. This was repeated several times until the desired amount of sulphur was infiltrated. The loading density of sulfur was approximately $2.0 \mathrm{mg} / \mathrm{cm}^{2}$ in HDCNT sample.The amount of sulphur in the cathode was measured 10 as the change of sample mass after the $S$ infiltration, and it was controlled by the concentration of sulphur solution and by the number of times that the infiltration process was repeated.

In this work, SEM was used to analyze the surface morphologies of the carbon materials before and after sulphur

15 loading. Two different SEM instruments (JEOL 5800 and FEG 6400) were used for scanning the samples. Energy dispersive $X$ ray spectroscopy (EDS) was carried out using a JEOL $5800 \mathrm{LV}$ scanning electron microscope (SEM) with an energy dispersive X-ray spectroscopy (EDS) detector. Transmission electron 20 microscope (TEM) (FEI Tecnai F20-G2 FEGTEM) was used for high resolution CNT characterizations. Raman data were obtained on a Dilor XY-800 spectrometer, using a $514 \mathrm{~nm}$ wavelength from an argon-ion laser.

\section{${ }_{25} 2.2 \mathrm{Li}-\mathrm{S}$ battery assembly and electrochemical measurements}

A Li-S battery was assembled in a glove box filled with pure argon. The same lithium metal foil (99.99\%, Sigma-Aldrich) was used as the anode and also as the reference electrode. The separator between the electrodes was a commercial microporous 30 polypropylene product (Celgard 2400). A copper foil was used as the anode current collector and the $\mathrm{Cu}$ foil that supported the CNT growth was used as the cathode current collector. The electrolyte in this study was based on $1 \mathrm{M} \mathrm{LiN}\left(\mathrm{CF}_{3} \mathrm{SO}_{2}\right)_{2}$ (99.95\%, trace metals basis, Sigma-Aldrich) dissolved in a 35 mixture of dioxolane (DOL) (99.8\%, Sigma-Aldrich) and 1,2dimethoxyethane (DME) (99.5, Sigma-Aldrich) in a volume ratio of $1: 1$. The galvanostatic charge/discharge tests were performed at a specific current of $400 \mathrm{~mA} \mathrm{~g}^{-1}$ of $\mathrm{S}$ to evaluate the electrochemical capacity and cycle life of the electrodes at room 40 temperature using a LAND-CT2001A instrument (Wuhan, China). The cut-off potentials for charge and discharge were set

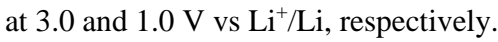

\section{Theory/Calculation}

\section{${ }_{45}$ 3.1 Computational simulation of the sulphur adsorption.}

The generalized gradient approximation (GGA) with the PerdewBurke-Ernzerhof (PBE) functional[39] was employed to describe the exchange and correlation effects. The DFT + D method within the TS scheme[40] was used in all the calculations to 50 consider the van der Waals forces. Vanderbilt ultrasoft pseudopotential, plane wave basis set with $330 \mathrm{eV}$ cutoff energy, and Monkhorst-Pack k point meshes as dense as $0.025 \AA^{-1}$ in each orthogonal direction were used. The convergence tolerance of energy of $10^{-5} \mathrm{eV}$ was taken, and the maximal allowed force and 55 displacement were $0.05 \mathrm{eV} \AA^{-1}$ and $0.002 \AA$, respectively. A small nanotube $(6,6)$ was used due to limited computational resources.

\section{Results and discussion}

The CNTs synthesized in this work are typically $\sim 100-250 \mu \mathrm{m}$ in 60 length and vertically aligned (Fig. s1a). Both the individual CNTs and the empty space between them can be clearly seen in Fig. 1a and s1b. The CNTs typically have 2-5 walls (the number of carbon shells of a CNT) with closed end at the tip [35, 41] and with an average diameter of $\sim 7 \mathrm{~nm}$ (Fig. s1 c, e and f). The ${ }_{65}$ Raman spectrum of CNT in Fig. s1d shows a G/D peak ratio of 1.5, which demonstrates the good crystalline quality of the tubes.[42] We note that the CNTs provide a good electronic conduction path and also can facilitate ion transport within the electrolyte, and the empty space between the tubes can 70 accommodate the sulphur infiltrated into the CNT forest.

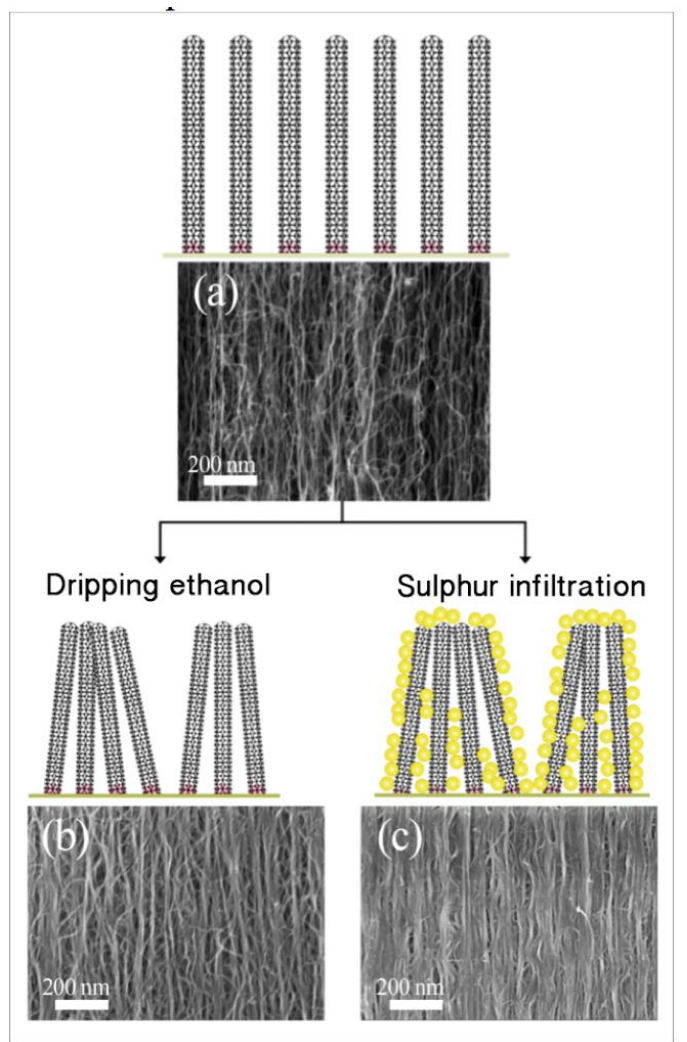

Fig.1 Schematic and the corresponding SEM images of carbon nanotubes. (a) as-grown forest, (b) after infiltration by ethanol. (c) after sulphur infiltration. 

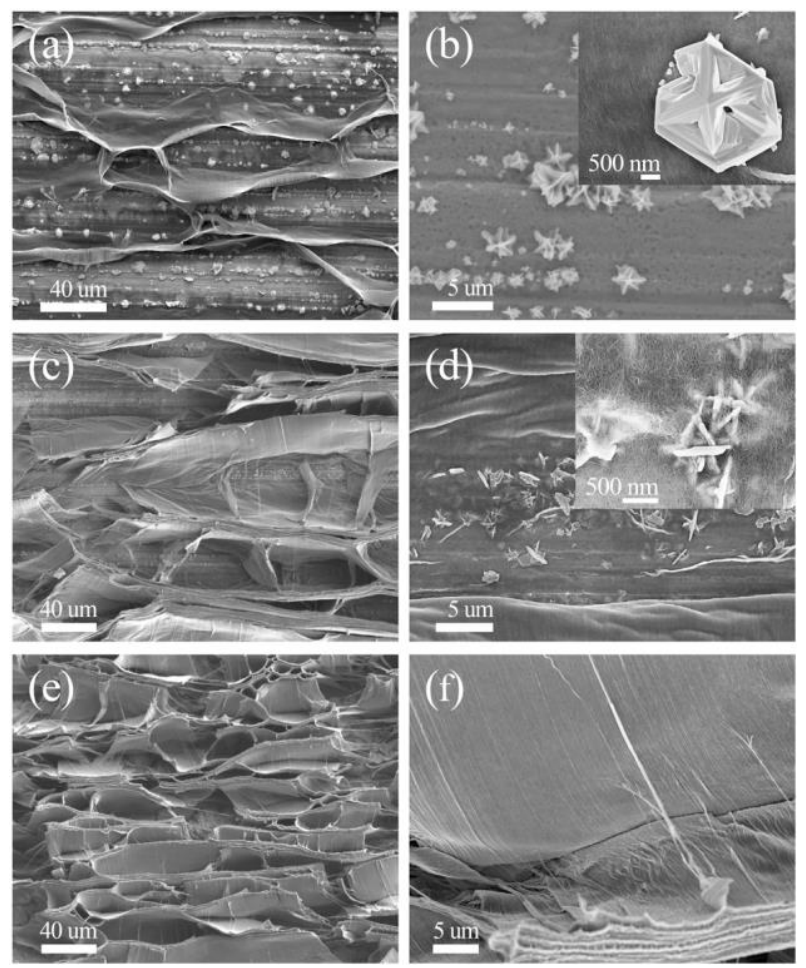

Fig.2 SEM images of CNT forest after S infiltration. (a) and (b) low density (LD) CNT, with 59 wt\% sulphur. (c) and (d) median density (MD) CNT, with 63 wt\% sulphur. (e) and (f) high density (HD) CNT, with 62 wt\% sulphur. (b) 5 Its inset show the relatively large amount of $S$ crystals exposed on the substrate surface. (d) shows that there is a much smaller amount of $\mathrm{S}$ exposed and most $\mathrm{S}$ crystals are now covered by the close-packed CNT network. (f) shows that no $S$ crystals are observed and nearly all $S$ content is now locked into the close-packed CNTs.

10 The schematics of the sulphur infiltration are shown in Fig. 1.

Fig. 1 (a) shows the structure of vertical aligned CNT forest that was used for infiltrating the elemental sulphur. By comparing the CNTs after ethanol (Fig. 1b)[33, 34] and sulphur [30] (Fig. 1c) infiltration, it is very clear that the CNT bundles with $\mathrm{S}$ 15 infiltration are larger in diameter and appear to be packed more closely. The difference is caused by a homogenous coating of sulphur on the CNT sidewalls, as shown in Fig. 1c. The sulphur content is expected to be locked in the space between the tubes and this will physically prevent or limit the runoff of sulphur and ${ }_{20}$ polysulphides during the charge-discharge of the battery.

The sulphur infiltration process is greatly affected by the CNT forest density. Here, we define the CNT density as the percentage of area covered by the close-packed CNTs after the sulphur infiltration[34, 43-45]. This liquid-induced compaction 25 method enables a simple way to directly compare the CNT densities. After liquid sulphur is dropped, the CNT forest forms locally compacted regions by drying in air, which is caused by strong capillary forces during evaporation and strong vander waals interactions between condensed nanotubes. A larger 30 coverage by the close-packed CNTs means a higher areal density of the as-grown tubes on the substrate. Fig. s2 shows the SEM images of the top of a CNT forest before and after sulphur infiltration. The compacted area (red colour) is divided by the whole image area, yielding the fraction of compacted CNTs 35 (filling factor), showing in Fig s2b.

We have selected CNT forests of three different densities to investigate how the Li-S battery performance is affected by the CNT density. Fig. 2 shows the SEM images of the CNTs after S infiltration. The tube density in Fig 2a, c, e is 3\%, 11\% and $18 \%$ 40 respectively, and they are referred to as low density (LD), medium density (MD) and high density (HD) CNTs hereafter. These three CNT-S structures all have very similar S content of about 59-63 wt\%. However, for LD-CNTs, it is very clear that there are many $\mathrm{S}$ crystals exposed on the sample surface (Fig. 2a 45 and $2 \mathrm{~b}$ ). This is because that the close-packed CNT forest cannot accommodate most of the $\mathrm{S}$ content, and the $\mathrm{S}$ crystals are precipitated on the thin CNT layer which covers the substrate (Fig. 2b inset). For the MD-CNTs (Fig. 2c and 2d), a significantly larger percentage of the substrate area is covered by the close50 packed CNTs, and the CNTs can accommodate most of S content or at least partially cover the $\mathrm{S}$ crystals precipitated on the substrate surface (Fig. 2d and inset). In the case of HD-CNTs, in Fig. 2(e, f), no $S$ crystals are observed under similar magnifications. This is attributed to the efficient soaking of the S55 containing solution by the CNT forest and the subsequently uniform locking of the S into the close-packed CNT forest.

Elemental mapping was used to evaluate the uniformity of the carbon and sulphur distribution in the HD-CNT-S composite. Fig. 3 (b, c) shows the S-rich regions overlapping with the C-rich 60 regions. This confirms that $\mathrm{S}$ is uniformly distributed between the neighboring CNTs (Fig. 2f). In the regions that have less coverage by the CNTs, the sulphur loading becomes less and this region overlaps with the $\mathrm{Cu}$-rich regions which represent the metal substrate (Fig. 3d). We also note that the amount of $\mathrm{S}$ ${ }_{65}$ incorporated into the CNTs can be controlled by the $\mathrm{S}$ concentration in the solution or number of the drops dipped on the CNTs. In terms of MD-CNT-S and LD-CNT-S composites, an inhomogeneous sulphur distribution could be observed obviously from EDS mapping in Fig. s3 and s4.

70
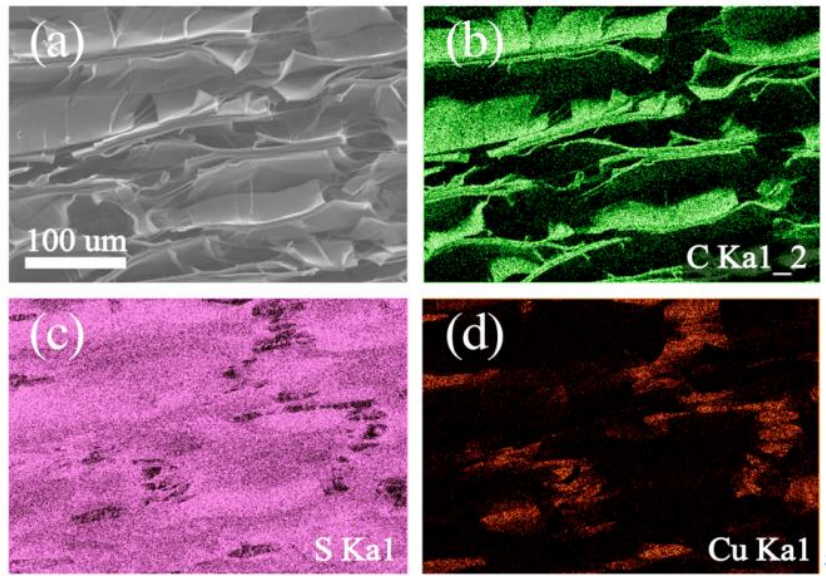

Fig.3 SEM image and elemental maps of (b) carbon, (c) sulphur, and (d) copper in the HD-CNT-S composites with $62 \mathrm{wt} \%$ sulphur.

Fig. 4a shows the initial galvanostatic discharge curves for 75 cells from the as-prepared S/CNT samples. It is clear that the S/HD-CNT cathode shows a higher initial discharge capacity and higher flat voltage plateau as compared with other two samples. It demonstrates two potential regions, one sloping down from $2.4-$ $2.1 \mathrm{~V}$ (vs. Li/ $/ \mathrm{Li}^{+}$), corresponding to the formation of long-chain 80 soluble lithium polysulphides $\left(\mathrm{Li}_{2} \mathrm{~S}_{\mathrm{n}}\right.$; where $\mathrm{n}$ is typically $\left.4-8\right)$ 
and a second region as a plateau at $2.1 \mathrm{~V}$ corresponding to shortchain solid sulphides $\left(\mathrm{Li}_{2} \mathrm{~S}_{2}\right.$ and $\left.\mathrm{Li}_{2} \mathrm{~S}\right)$. [3, 6, 46, 47] For the sulphur/MD-CNT and sulphur/LD-CNT arrays, the upper potential regions sharply decline corresponding to very low $\mathrm{S}$ 5 utilization. The clear difference of the electrochemical behaviour in the $1^{\text {st }}$ discharge cycle of both MD- and LD-CNT based cathodes can be attributed to a number of factors, such as existence of bulk insulating sulphur and high ohmic polarization with these two CNT-S composites. These could be attributed to 10 non-uniform sulphur loading for the LD- and MD-CNT arrays which also contain numerous and large proportion of electrochemically ineffective porous space (Fig. 2a, c). Sulphur agglomerates that are exposed outside of the CNTs could lead to reduction in electrical conductivity, giving rise to lower potential 15 plateau. The cathode based on HD-CNTs is effective in achieving $80 \%$ of theoretical capacity in the $1^{\text {st }}$ cycle with good utilization of $\mathrm{S}$ in both the high potential and the lower plateau regions. Only beyond $1000 \mathrm{mAh} \mathrm{g}{ }^{-1}$, loss in potential, possibly due to ohmic polarization is visible. Thus it can be hypothesized that the 20 HD-CNT vertical arrays provide a suitable network for uniformly distributing sulphur in the CNT electrode surfaces (for improving triple phase boundaries) and for holding the polysulphides in the cathode nanopores for electrochemical utilization rather than encouraging loss by diffusion.
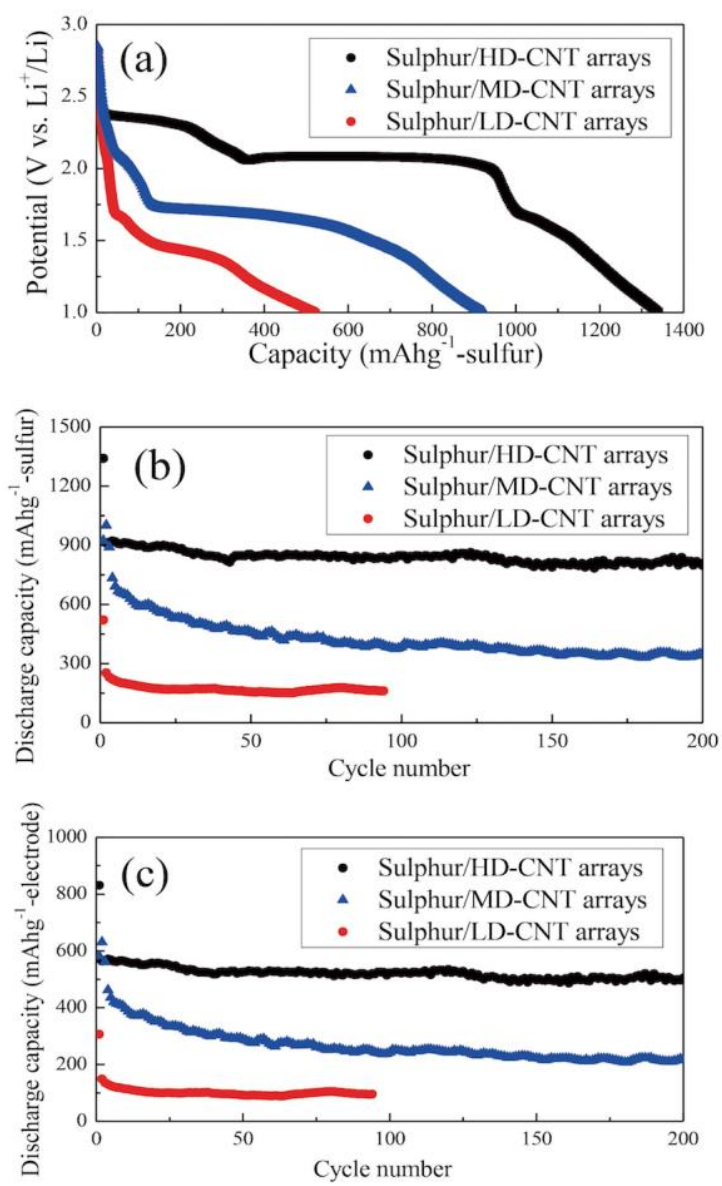

25

Fig.4 The first discharge curves and (b, c) cycle performance of the samples at a specific current of $400 \mathrm{~mA} \mathrm{~g}^{-1}$ of $\mathrm{S}$ between 1.0 and $3.0 \mathrm{~V}$ (vs $\left.\mathrm{Li}^{+} / \mathrm{Li}\right)$ in the electrolyte $\left(\mathrm{LiN}\left(\mathrm{CF}_{3} \mathrm{SO}_{2}\right)_{2} / \mathrm{DOL}: \mathrm{DME}=1: 1(\mathrm{v} / \mathrm{v})\right)$. The weight percentage of the $\mathrm{S}$ loading in the cathode materials are $62 \%, 63 \%$ and
$3059 \%$ respectively for HD-CNT, MD-CNT and LD-CNT.

The cycle performance of the cells is shown in Fig. $4 \mathrm{~b}$ and Fig. 4c. The sulphur/HD-CNT has the best cycle performance of the three samples, as anticipated. It shows the highest initial discharge capacity of $1340 \mathrm{mAh} \mathrm{g}^{-1}$ of S $(80 \%$ of the theoretical 35 capacity) and a reversible capacity of $910 \mathrm{mAh} \mathrm{g}^{-1}$ of $\mathrm{S}$ in the second cycle, corresponding to impressive values of 831 and 564 $\mathrm{mAh} \mathrm{g}^{-1}$ of the cathode mass. The irreversible capacity loss in the first cycle is due to unembedded polysulphide species which might diffuse throughout the electrode and deposit on the lithium 40 side where they are reduced. After the second cycle, the average fading rate is small at $0.054 \%$ per cycle and the capacity was stabilized to a constant value at $503 \mathrm{mAh} \mathrm{g}^{-1}$ of the cathode mass (corresponding to $812 \mathrm{mAh} \mathrm{g}^{-1}$ of S) after the $200^{\text {th }}$ cycle. This stable capacity over long cycles can be attributed to the uniform 45 distribution of lithium polysulphides that are appropriately locked in the HD-CNT structures (Fig. 3c). Thus even after 200 cycles, nearly $50 \%$ of $\mathrm{S}$ participates in the electrochemical redox reactions. Long cycle life utilization of $50 \%$ of the loaded sulphur can still provide impressive capacity thus taking practical 50 application of Li-S battery further forward. Dramatically, the average decay rate seems to decrease as the cycle number increases, thus a steady state is likely to be achieved with respect to sulphur utilization in the discharge cycle and sulphur formation during charging. Addtionally, the sulphur/HD-CNT yields an 55 average columbic efficiency of $\sim 95 \%$, which is superior to that of sulphur/MD-CNT( $\sim 77 \%)$ and sulphur/LD-CNT( $\sim 82 \%)$ cathodes.
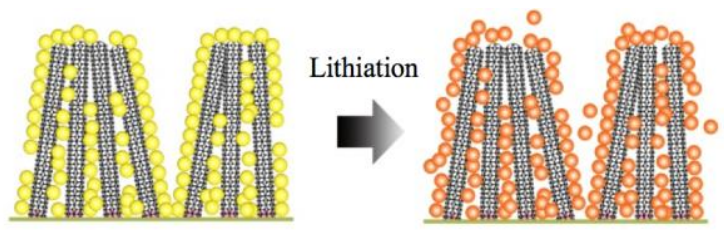

Effective trapping of polysulphides

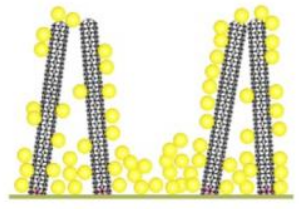

Sulphur

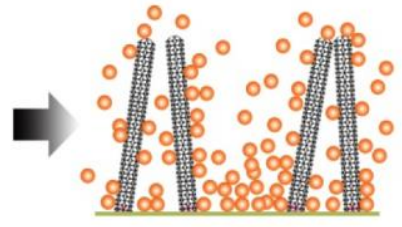

Dissolution of polysulphides

\section{Polysulphides}

Fig.5 Schematic of the lithiation process in Sulphur/CNT array composites

60 The mechanism of the polysulphide stabilization by the CNT scaffold is shown schematically in Fig. 5. The CNTs provide a good conductive path for current transport, and the flexible nature of the CNTs and the empty space between the tubes can accommodate the volume expansion of sulphur during lithiation.

${ }_{65}$ It should note that HD-CNTs can have more tolerance to the volume changes. These will result in an effective trapping of polysulphide during the charge and discharge cycles. In the case of LD-CNTs, there is excess empty space that is not covered by CNTs, and this will result in undesirable precipitation of large $\mathrm{S}$ 70 crystals on the substrate (Fig. 2b), which is not-conducive to generating triple phase boundaries (TPBs). 

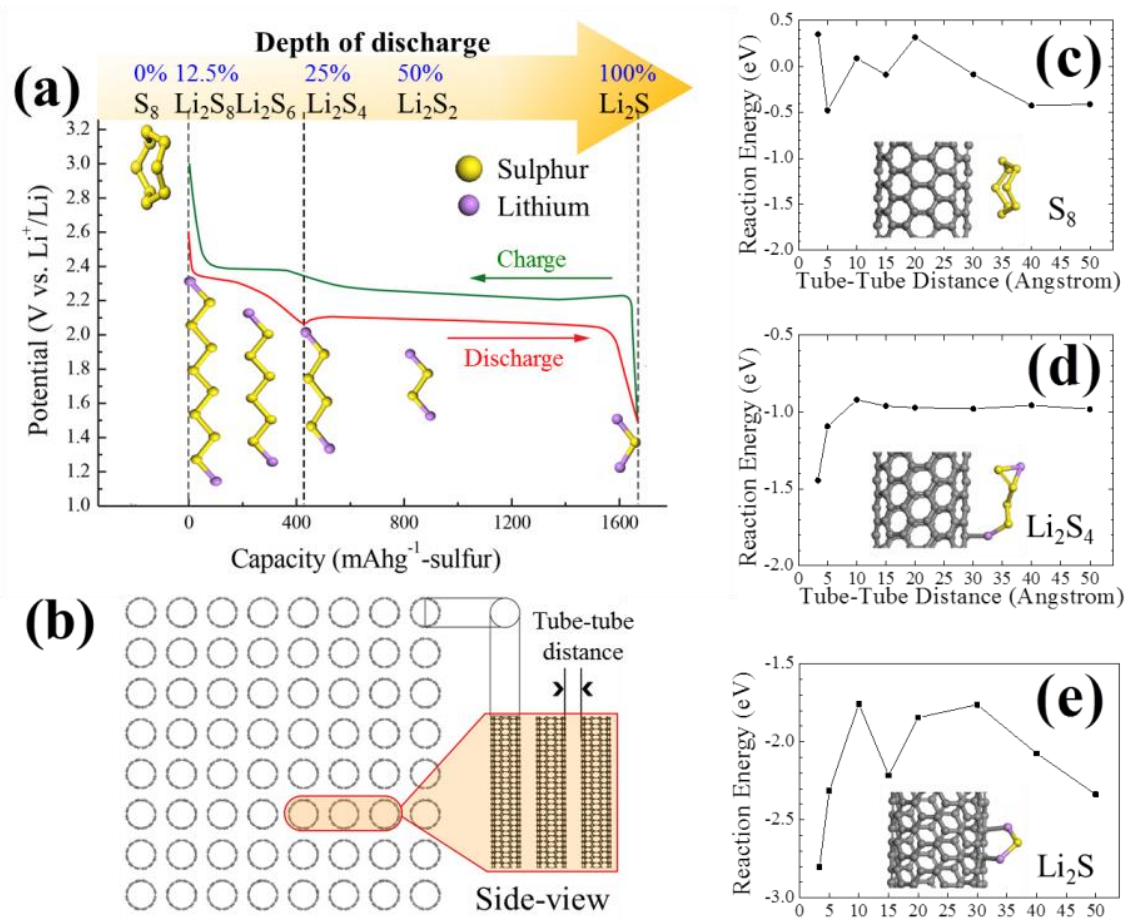

Top-view

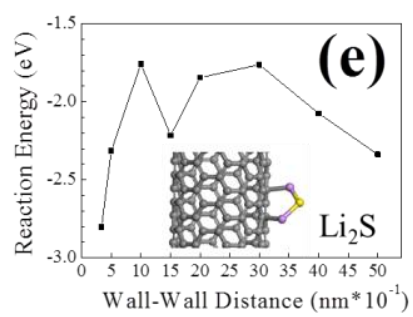

Fig.6. (a) The discharge-charge voltage profile and chemistry of sulphur cathode in the organic electrolyte. (b) carbon nanotube arrays used in the simulation. $\mathrm{S}_{8}(\mathrm{c}), \mathrm{Li}_{2} \mathrm{~S}_{4}(\mathrm{~d}), \mathrm{Li}_{2} \mathrm{~S}(\mathrm{e})$ adsorptions on $(6,6)$ single wall nanotube.

The insulating nature of sulphur and non-effective locking by the CNTs lead to a low utilization of active material sulphur and encourage diffusion following chemical dissolution of the polysulphides. These factors will cause both a low discharge 5 capacity and poor cycle performance of the cathodes. Additionally, cathodes are formulated normally using sulphur composite powder samples. These composite powders contain many interfaces and grain boundaries, resulting in several irregularly connected carbon networks. These interfaces act as 10 scattering centres for electron transport, leading to increased internal resistances of the cathode of a Li-S battery. Therefore, a high density CNT forest can lead to efficient polysulphide stabilization, enhanced TPBs and optimal sulphur loading which are crucial to produce higher specific capacity Li-S batteries with 15 long cycle life.

To study the nanotube density dependent absorbability of $S_{8}$, $\mathrm{Li}_{2} \mathrm{~S}_{4}$ (representing one of the series of lithium polysulphide intermediates) and $\mathrm{Li}_{2} \mathrm{~S}$, we carried out first-principles calculation by the CASTEP code.[48] We simulated the 20 adsorption of $\mathrm{Li}_{\mathrm{x}} \mathrm{S}_{\mathrm{y}}$ complex on nanotube in a tetragonal supercell structure; and we were able to sample a series of nanotube area densities by adjusting the supercell lattice constant.

We first studied $S_{8}$ adsorption on SWNT as shown in Fig. 6c, and then we sampled the smallest tube-to-tube distance with 3.34, ${ }_{25} 5.00, \quad 10.00, \quad 15.00,20.00, \quad 30.00, \quad 40.00,50.00 \AA$. The absorbability is evaluated by the reaction energy, which is defined as:

Reaction Energy $=\mathrm{E}(\mathrm{SWNT}+\mathrm{A})-\mathrm{E}(\mathrm{SWNT})-\mathrm{E}(\mathrm{A})$

where $\mathrm{E}(\mathrm{SWNT}+\mathrm{A})$ is the total energy of SWNT and adsorbate in
30 equilibrium adsorption, E(SWNT) the energy of isolated SWNT and $\mathrm{E}(\mathrm{A})$ the energy of isolated adsorbate.

A larger negative reaction energy represents a stronger absorbtion. As Fig. 6c shows, at the smallest tube-to-tube distance of $3.34 \AA$, which is the interlayer distance of carbon 35 layers in graphite, $\mathrm{S}_{8}$ is more resistant to adsorption compared with $\mathrm{Li}_{2} \mathrm{~S}_{4}$ and $\mathrm{Li}_{2} \mathrm{~S}$ in Fig. $6 \mathrm{~d}$ and e, respectively[49]. This is understandable by the large ring diameter of S8 (4.7 $\AA$ ). $\mathrm{S}_{8}$ would undergo a significant compression when being confined by the dense SWNT forest. An increase of the tube-to-tube distance to ${ }_{40} 5.00 \AA$ is expected to release the compression to some extent and make $\mathrm{S}_{8}$ more absorbable. Further increase in the distance, however, makes $\mathrm{S}_{8}$ more resistant to adsorption until the distance reaches $20.00 \AA$, when the adsorption starts to increase again. Generally, $\mathrm{S}_{8}$ keeps a significant distance of $\sim 3.00 \AA$ to the ${ }_{45}$ SWNT and forms weak non-covalent bond with the CNTs, therefore the absorbability is not strong, as shown in the inset of Fig. 6c. For $\mathrm{Li}_{2} \mathrm{~S}_{4}$ adsorption on a SWNT as shown in Fig. 6d, $\mathrm{Li}_{2} \mathrm{~S}_{4}$ forms $\mathrm{Li}-\mathrm{C}$ bond with SWNT at one end of the molecule chain with a bond length of about $2.20 \AA$, and the other end of the 50 chain is slightly distorted by forming a three-membered $\mathrm{LiS}_{2}$ ring, this is due to the long and soft chain structure of $\mathrm{Li}_{2} \mathrm{~S}_{4}$. As Fig. $6 \mathrm{~d}$ shows, very dense SWNT forest is favoured for the adsorption of $\mathrm{Li}_{2} \mathrm{~S}_{4}$. The reaction energy of $\mathrm{Li}_{2} \mathrm{~S}_{4}$ is more negative than that of $\mathrm{S}_{8}$ over the whole range of tube-to-tube distance. For $\mathrm{Li}_{2} \mathrm{~S}$ 55 adsorption on the same SWNT as shown in Fig. $6 \mathrm{e}, \mathrm{Li}_{2} \mathrm{~S}$ tends to form Li-C bond with SWNT with a bond length about $2.30 \AA$ A. It is clear that dense nanotubes are also energetically favorable for the adsorption of the sulphur compounds, similar to Fig. c,d. $\mathrm{Li}_{2} \mathrm{~S}$ 
is most absorbable in the smallest tube-to-tube distance of $3.34 \AA$ and maintains a large negative reaction energy (below $-1.7 \mathrm{eV}$ ) upto $50.00 \AA$.

Therefore, our calculations show that a small wall-to-wall 5 distance is favored to achieve large negative reaction energy of the adsorption. This is supported by experimental results which demonstrate that a high density nanotube forest is favored to achieve stronger $\mathrm{S}$ adsorption and improves the battery cycle life.

\section{Conclusions}

10 In summary, binder-free carbon nanotube forests of different densities have been used to fabricate the S/CNT cathodes for Li-S batteries. By using the HD-CNT arrays, the initial specific capacities of $1340 \mathrm{mAh} \mathrm{g}^{-1}$ and $831 \mathrm{mAh} \mathrm{g}^{-1}$ (of sulphur and electrode mass, respectively) are achieved. And the discharge 15 capacities of 812 and $503 \mathrm{mAh} \mathrm{g}^{-1}$ (of sulphur and electrode mass, respectively) are demonstrated after the $200^{\text {th }}$ cycle, with a capacity decay of only $0.054 \%$ per cycle. The good electrochemical performance could be attributed to the developed high-density CNT forest scaffolds supporting optimal sulphur 20 loading, adsorption of polysulphides and accommodating the volume expansion of sulphur in the basic organic ether eletrolyte, as supported by both experimental results and computational simulations.

The knowledge gained from this study is not only useful for 25 the design of efficient new electrode structure, but also for understanding the effect of the density of CNTs on the electrochemical cycle stability. This work can be applied to other high energy density electrode systems, especially to those that undergo the active material dissolution and volume expansion 30 during redox reactions.

\section{Acknowledgements}

Kai Xi wishes to thank the Cambridge Overseas Trust. The authors would like to thank Guoran Li and Yanjun Liu (Central ${ }_{35}$ Saint Martins - University of the Arts London) for their assistance in preparing the paper for publication.

\section{Appendix A. Supporting information}

${ }_{40}$ Supplementary data associated with this article can be found in the online version at ...

\section{References}

45 [1] S. Evers, L.F. Nazar, Accounts of Chemical Research, 46 (2012) 11351143.

[2] P.G. Bruce, S.A. Freunberger, L.J. Hardwick, J.-M. Tarascon, Nat Mater, 11 (2012) 19-29.

[3] Y. Yang, G.Y. Zheng, Y. Cui, Chem. Soc. Rev., 42 (2013) 3018-3032.

50 [4] A. Manthiram, Y. Fu, Y.-S. Su, Accounts of Chemical Research, 46 (2012) 1125-1134.

[5] R.D. Rauh, K.M. Abraham, G.F. Pearson, J.K. Surprenant, S.B. Brummer, J. Electrochem. Soc., 126 (1979) 523-527.

[6] J. Shim, K.A. Striebel, E.J. Cairns, J. Electrochem. Soc., 149 (2002) 55 A1321-A1325.

[7] X. Ji, K.T. Lee, L.F. Nazar, Nat Mater, 8 (2009) 500-506.
[8] H.L. Wang, Y. Yang, Y.Y. Liang, J.T. Robinson, Y.G. Li, A. Jackson, Y. Cui, H.J. Dai, Nano Lett., 11 (2011) 2644-2647.

[9] B. Zhang, X. Qin, G.R. Li, X.P. Gao, Energy \& Environmental 60 Science, 3 (2010) 1531-1537.

[10] K. Xi, S. Cao, X. Peng, C. Ducati, R. Vasant Kumar, A.K. Cheetham, Chemical Communications, 49 (2013) 2192-2194.

[11] K. Xi, P.R. Kidambi, R. Chen, C. Gao, X. Peng, C. Ducati, S. Hofmann, R.V. Kumar, Nanoscale, (2014).

65 [12] M.-Q. Zhao, Q. Zhang, J.-Q. Huang, G.-L. Tian, J.-Q. Nie, H.-J. Peng, F. Wei, Nat Commun, 5 (2014).

[13] G. Zheng, Q. Zhang, J.J. Cha, Y. Yang, W. Li, Z.W. Seh, Y. Cui, Nano Lett., 13 (2013) 1265-1270.

[14] X.L. Ji, S. Evers, R. Black, L.F. Nazar, Nat. Commun., 2 (2011).

70 [15] R. Demir-Cakan, M. Morcrette, F. Nouar, C. Davoisne, T. Devic, D. Gonbeau, R. Dominko, C. Serre, G. Ferey, J.M. Tarascon, J. Am. Chem. Soc., 133 (2011) 16154-16160.

[16] S. Evers, T. Yim, L.F. Nazar, The Journal of Physical Chemistry C, 116 (2012) 19653-19658.

75 [17] Y. Fu, Y.-S. Su, A. Manthiram, ACS Applied Materials \& Interfaces, 4 (2012) 6046-6052.

[18] Z. Wei Seh, W. Li, J.J. Cha, G. Zheng, Y. Yang, M.T. McDowell, P.-C. Hsu, Y. Cui, Nat Commun, 4 (2013) 1331.

[19] G.-C. Li, G.-R. Li, S.-H. Ye, X.-P. Gao, Advanced Energy Materials, 802 (2012) 1238-1245.

[20] C. Huang, J. Xiao, Y. Shao, J. Zheng, W.D. Bennett, D. Lu, L.V. Saraf, M. Engelhard, L. Ji, J. Zhang, X. Li, G.L. Graff, J. Liu, Nat Commun, 5 (2014).

[21] J.-Q. Huang, Q. Zhang, H.-J. Peng, X.-Y. Liu, W.-Z. Qian, F. Wei, 85 Energy \& Environmental Science, 7 (2014) 347-353.

[22] W. Weng, V.G. Pol, K. Amine, Advanced Materials, 25 (2013) 16081615.

[23] L. Suo, Y.-S. Hu, H. Li, M. Armand, L. Chen, Nat Commun, 4 (2013) 1481.

90 [24] E.S. Shin, K. Kim, S.H. Oh, W.I. Cho, Chemical Communications, 49 (2013) 2004-2006.

[25] D. Aurbach, E. Pollak, R. Elazari, G. Salitra, C.S. Kelley, J. Affinito, J. Electrochem. Soc., 156 (2009) A694-A702.

[26] S.C. Han, M.S. Song, H. Lee, H.S. Kim, H.J. Ahn, J.Y. Lee, J. 95 Electrochem. Soc., 150 (2003) A889-A893.

[27] J.C. Guo, Y.H. Xu, C.S. Wang, Nano Lett., 11 (2011) 4288-4294.

[28] J.J. Chen, Q. Zhang, Y.N. Shi, L.L. Qin, Y. Cao, M.S. Zheng, Q.F. Dong, Phys. Chem. Chem. Phys., 14 (2012) 5376-5382.

[29] S. Doerfler, M. Hagen, H. Althues, J. Tuebke, S. Kaskel, M.J. 100 Hoffmann, Chemical Communications, 48 (2012) 4097-4099.

[30] M. Hagen, S. Dorfler, H. Althues, J. Tubke, M.J. Hoffmann, S. Kaskel, K. Pinkwart, J. Power Sources, 213 (2012) 239-248.

[31] R. Chen, T. Zhao, J. Lu, F. Wu, L. Li, J. Chen, G. Tan, Y. Ye, K. Amine, Nano Lett., 13 (2013) 4642-4649.

105 [32] G. Zhou, D.-W. Wang, F. Li, P.-X. Hou, L. Yin, C. Liu, G.Q. Lu, I.R. Gentle, H.-M. Cheng, Energy \& Environmental Science, 5 (2012) 8901 8906.

[33] C. Zhang, R. Xie, B. Chen, J. Yang, G. Zhong, J. Robertson, Carbon, 53 (2013) 339-345.

110 [34] G.F. Zhong, J.H. Warner, M. Fouquet, A.W. Robertson, B.A. Chen, J. Robertson, Acs Nano, 6 (2012) 2893-2903.

[35] G. Zhong, S. Hofmann, F. Yan, H. Telg, J.H. Warner, D. Eder, C. Thomsen, W.I. Milne, J. Robertson, J Phys Chem C, 113 (2009) 1732117325.

115 [36] C. Zhang, F. Yan, B.C. Bayer, R. Blume, M.H. van der Veen, R.S. Xie, G.F. Zhong, B.G. Chen, A. Knop-Gericke, R. Schlogl, B.D. Capraro, S. Hofmann, J. Robertson, J Appl Phys, 111 (2012).

[37] S. Esconjauregui, M. Fouquet, B.C. Bayer, C. Ducati, R. Smajda, S. Hofmann, J. Robertson, Acs Nano, 4 (2010) 7431-7436.

120 [38] B. Chen, P.G. Oppenheimer, T.A.V. Shean, C.T. Wirth, S. Hofmann, J. Robertson, Journal of Physical Chemistry C, 116 (2012).

[39] J.P. Perdew, K. Burke, M. Ernzerhof, Physical Review Letters, 77 (1996) 3865-3868

[40] A. Tkatchenko, M. Scheffler, Physical Review Letters, 102 (2009).

125 [41] A.M. Cassell, J.A. Raymakers, J. Kong, H. Dai, The Journal of Physical Chemistry B, 103 (1999) 6484-6492.

[42] R. Xie, C. Zhang, M.H. van der Veen, K. Arstil, T. Hantschel, B. Chen, G. Zhong, J. Robertson, Nanotechnology, 24 (2013) 125603.

[43] D.N. Futaba, K. Hata, T. Yamada, T. Hiraoka, Y. Hayamizu, Y. 
Kakudate, O. Tanaike, H. Hatori, M. Yumura, S. Iijima, Nature Materials, 5 (2006) 987-994.

[44] N. Chakrapani, B.Q. Wei, A. Carrillo, P.M. Ajayan, R.S. Kane, P Natl Acad Sci USA, 101 (2004) 4009-4012.

5 [45] S. Kaur, S. Sahoo, P. Ajayan, R.S. Kane, Advanced Materials, 19 (2007) 2984-+.

[46] Y.V. Mikhaylik, J.R. Akridge, J. Electrochem. Soc., 151 (2004) A1969-A1976

[47] X. Ji, L.F. Nazar, Journal of Materials Chemistry, 20 (2010) 9821 109826.

[48] M.D. Segall, P.J.D. Lindan, M.J. Probert, C.J. Pickard, P.J. Hasnip, S.J. Clark, M.C. Payne, Journal of Physics-Condensed Matter, 14 (2002) 2717-2744.

[49] Y. Yang, M.T. McDowell, A. Jackson, J.J. Cha, S.S. Hong, Y. Cui, 15 Nano Lett., 10 (2010) 1486-1491. 